\title{
A review of antenatal corticosteroid use in premature neonates in a middle-income country
}

\author{
A Laher, ${ }^{1} \mathrm{MB}$ BCh, DCH (SA); D E Ballot, ${ }^{1} \mathrm{MB}$ BCh, FCPaed (SA), PhD; T Ramdin, ${ }^{1} \mathrm{MB}$ BCh, FCPaed (SA), DCH (SA), \\ MMed (Neonatology) (SA); T Chirwa, ${ }^{2}$ BSc, MSc, PG Dip, PhD \\ ${ }^{1}$ Department of Paediatrics and Child Health, Faculty of Health Sciences, University of the Witwatersrand, Johannesburg, South Africa \\ ${ }^{2}$ School of Public Health, Faculty of Health Sciences, University of the Witwatersrand, Johannesburg, South Africa
}

Corresponding author: A Laher (anees.laher@gmail.com)

Background. Antenatal corticosteroid (ANS) use in premature neonates has become a standard of practice. However, there is low ANS coverage in low- to middle-income countries (LMICs). Recent studies have questioned the efficacy of ANSs in such countries.

Objective. To review the use of ANSs in preterm neonates at Charlotte Maxeke Johannesburg Academic Hospital (CMJAH), South Africa. Methods. This was a retrospective observational study of all neonates with a birth weight of $500-1800 \mathrm{~g}$ born at CMJAH between 1 January 2013 and 30 June 2016. Neonatal and maternal characteristics of neonates exposed to ANSs were compared with those of neonates who were not exposed.

Results. The ANS coverage of the final sample was 930/2 109 (44.1\%). The mean (standard deviation (SD)) birth weight was 1292.4 (323.2) $\mathrm{g}$ and the mean gestational age 30.2 (2.9) weeks. Attending antenatal care and maternal hypertension were associated with increased use of ANSs, whereas vaginal delivery was associated with decreased use. In neonates weighing $<1500 \mathrm{~g}$, the use of ANSs was associated with decreased mortality, decreased intraventricular haemorrhage and decreased patent ductus arteriosus. There was no association between ANSs and respiratory distress syndrome, necrotising enterocolitis, sepsis or need for respiratory support in all premature neonates, and no association with improved outcomes in those weighing $\geq 1500 \mathrm{~g}$.

Conclusion. The benefits of ANSs in terms of neonatal morbidity in this study were not as marked as those published in high-income countries. A randomised controlled trial may be indicated in LMICs.

S Afr Med J 2017;107(9):768-772. DOI:10.7196/SAMJ.2017.v107i9.12246

Neonatal mortality is a major problem worldwide, accounting for $38 \%$ of all deaths in children aged $<5$ years. ${ }^{[1]}$ Every year, it is estimated that four million babies die in the neonatal period. ${ }^{[1]}$ Preterm birth is the most common cause of neonatal mortality, accounting for $28 \%$ of neonatal deaths. ${ }^{[1]}$ Of great concern, $99 \%$ of neonatal deaths occur in low-income countries, with poverty being strongly associated with an increased risk of neonatal mortality. ${ }^{[1]}$

Africa accounts for $28 \%$ of neonatal deaths worldwide. ${ }^{[1]}$ South Africa (SA) has had some success in reducing childhood mortality in recent years, but has not met the fourth Millennium Development Goal of reducing mortality by two-thirds in children aged $<5$ years. ${ }^{[2]}$ SA is one of the countries in which neonatal mortality has not decreased over the past 20 years. ${ }^{[3]}$

Addressing neonatal mortality is of vital importance in reducing childhood mortality. Specifically, reducing mortality in preterm neonates can be the most beneficial, as prematurity is the most common cause of neonatal mortality. ${ }^{[1]}$

In 1972, Liggins and Howie ${ }^{[4]}$ conducted the first trial using antenatal corticosteroids (ANSs) for the prevention of respiratory distress syndrome (RDS) in preterm neonates. They noted a reduction in the incidence of RDS in preterm neonates whose mothers were given ANSs compared with preterm neonates whose mothers were not. ${ }^{[4]}$ Since this landmark trial, ${ }^{[4]}$ ANS therapy has become a standard of care to prevent preterm neonatal complications. ${ }^{[5]}$

A Cochrane review of 21 articles evaluating the effect of ANSs on preterm neonates ${ }^{[5]}$ showed that ANSs reduced the risk of RDS, intraventricular haemorrhage (IVH), necrotising enterocolitis (NEC) and early-onset sepsis, reduced mortality and the need for respiratory support, and resulted in a trend towards reduced need for surfactant therapy. However, of these 21 studies, 17 were conducted in highincome countries and only 4 in middle-income countries. ${ }^{[5]}$ In addition to the benefits shown in the Cochrane review, there seems to be a beneficial effect of ANSs on symptomatic patent ductus arteriosus (PDA) ${ }^{[6]}$

The use of ANSs in low- and middle-income countries (LMICs) remains low. ${ }^{[7]}$ The main reason is mothers being admitted in advanced labour. ${ }^{[8]}$ Other reasons include inevitable abortion, obstetric emergencies necessitating immediate delivery, incorrect assessment of gestational age, refusal of hospital treatment, and obstetricians missing an opportunity to provide mothers with ANSs. ${ }^{[8]}$ The latter should decrease, as the National Institute for Health and Care Excellence recommends routine administration of ANSs to women who are in suspected or established preterm labour at a gestational age $<34$ weeks. ${ }^{[9]}$

A recent article by Azad and Costello ${ }^{[10]}$ suggests that caution is needed before increasing the use of ANSs in LMICs. They question the efficacy of ANSs in LMICs to improve mortality in premature neonates at a gestational age of $<33$ weeks, and state that ANSs are of no benefit after 33 weeks' gestation. ${ }^{[10]}$

Althabe et al. ${ }^{[1]}$ conducted a randomised trial in six LMICs (Argentina, Guatemala, India, Kenya, Pakistan and Zambia) to implement strategies to reduce neonatal mortality. Strategies included identifying women at risk for preterm birth and upscaling ANSs in these women, whereas standard practice was continued in the control group. These strategies resulted in an increase in ANS coverage from $10 \%$ in the control group to $45 \%$ in the intervention group. ${ }^{[1]}$ Despite the increase in ANS coverage, the intervention group showed an increase in neonatal mortality, with an excess of 3.5 neonatal deaths 
for every 1000 women exposed to this strategy. ${ }^{[11]}$ It was not possible to assess the weight or gestational age of the babies in the study, and the cause of this increased mortality was not clear.

There is limited information on ANS use in SA. The Dexiprom study is the only study that has looked at effects of ANSs in SA, but it only involved mothers who had preterm premature rupture of membranes. ${ }^{[12]}$ There is concern over the use of ANSs in LMICs.

\section{Objective}

To review ANS use in a hospital in SA, a middle-income country.

\section{Methods \\ Study design}

This was a retrospective observational study. The study population included all neonates with a birth weight between $500 \mathrm{~g}$ and $1800 \mathrm{~g}$ born at Charlotte Maxeke Johannesburg Academic Hospital (CMJAH) between 1 January 2013 and 30 June 2016. Exclusion criteria included babies with lethal birth defects and those with unobtainable records. The total sample was analysed and then divided into two groups: babies weighing $\geq 1500 \mathrm{~g}$ and those weighing $<1500 \mathrm{~g}$. Each of the two groups was further divided into an ANS-exposed group and an unexposed group, and these groups were then compared. The ANS-exposed group were neonates whose mothers were given ANS, and the ANS-unexposed group were those whose mothers were not. At CMJAH, two doses of $12 \mathrm{mg}$ betamethasone 12 hours apart are given intramuscularly to mothers if the gestational age is $<34$ weeks or if the estimated fetal weight is $<2000$ g. In this study, birth weight was used instead of gestational age because gestational age is not accurate in our setting, where antenatal ultrasound is not routinely done and patients' recollection of the date of their last menstrual period is often inaccurate.

RDS is diagnosed using chest radiographs and clinically (tachypnoea, subcostal recessions, intercostal recessions) in premature neonates requiring oxygen at $\mathrm{CMJAH}$. The use of surfactant and continuous positive airway pressure (CPAP) is limited to neonates with a birth weight of $>750 \mathrm{~g}$, and is at the discretion of the attending doctor. Early rescue surfactant is used at CMJAH. The combination of early rescue surfactant and CPAP has been shown to reduce morbidity and mortality associated with intensive care unit admissions at CMJAH. ${ }^{[13]}$

\section{Data}

This was a secondary review of an existing database. Neonatal records are kept in a neonatal database at CMJAH. Data are managed using Research Electronic Data Capture (REDCap) ${ }^{[14]}$ which is hosted by the University of the Witwatersrand. Data are collected by clinicians using hospital records and verified at several different points. Permission was obtained to use this neonatal database.

\section{Statistical analysis}

Maternal and neonatal variables were evaluated. Valid cases were analysed and missing data excluded. Data were described using standard statistical methods. Categorical variables were described using frequencies and percentages, while continuous variables were described using means and standard deviations (SDs) or medians and interquartile ranges, depending on the distribution of the data. Chi-square tests were used to compare categorical variables. Student's independent $t$-test was used to compare continuous variables with a normal distribution. Non-parametric tests were used for skewed data. The level of significance was set at $p<0.05$. Analysis was done using SPSS, version 24 (IBM, USA). Associations with the use of ANSs were determined using logistic regression. All variables that approached significance $(p<0.06)$ on univariate analysis were explored.

\section{Ethical approval}

The study was approved by the Human Research Ethics Committee of the University of the Witwatersrand (ref. no. M160730).

\section{Results}

There was a total of 6186 neonates on the REDCap database (accessed on 27 September 2016) between 1 January 2013 and 30 June 2016. Of the 6186 neonates, 3415 had a birth weight $>1800 \mathrm{~g}$ and six a birth weight of $<500 \mathrm{~g}$. A further 656 were excluded (Fig. 1), resulting in a final sample size of 2109 . The mean (SD) birth weight of the final sample was 1292.4 (323.2) g, and the mean gestational age was 30.2 (2.9) weeks. ANS coverage of the final sample was less than half, with 930/2 109 neonates (44.1\%) exposed to ANSs and 1 179/2 109 (55.9\%) not exposed.

\section{Maternal characteristics}

Maternal characteristics are shown in Table 1. Mothers who did not attend antenatal care were significantly less likely to receive ANSs $(p<0.001)$. It was also observed that mothers with hypertension were more likely to receive ANSs $(p<0.001)$, whereas HIV-positive mothers were less likely $(p=0.001)$. There were $718 / 2101$ vaginal deliveries (34.2\%) and $1383 / 2101$ caesarean sections (65.8\%). Vaginal deliveries were associated with decreased ANS coverage $(p<0.001)$. There was no significant association between ANS coverage and maternal age, parity, chorioamnionitis or diabetes.

\section{Neonatal characteristics}

Neonatal characteristics of the total sample are shown in Table 2 and continuous variables in Table 3. Valid data are shown in the table and percentages are expressed in columns.

Gender, spontaneous gastrointestinal perforation, oxygen required by day 28 of life and home oxygen required were not included in Table 2, as none of these variables was significantly different. Reported associations with ANSs were included in the table, even though these variables were not significant in the current study.

\section{Total sample (Table 2)}

In the total sample, the variables late-onset sepsis $(p=0.017)$, RDS $(p<0.001)$, CPAP $(p=0.002)$ and surfactant therapy $(p=0.016)$ were

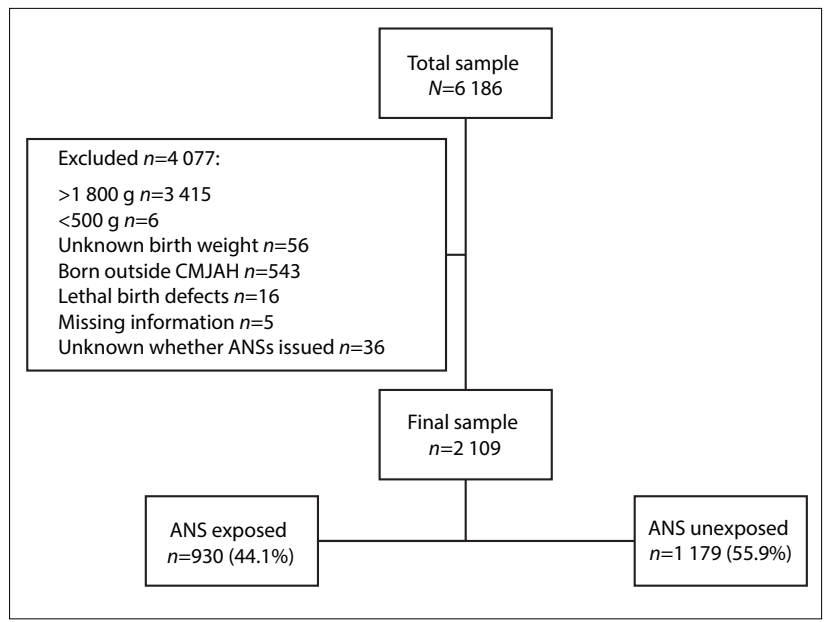

Fig. 1. Patient selection. (CMJAH = Charlotte Maxeke Johannesburg Academic Hospital; ANSs = antenatal steroids.) 
Table 1. Maternal characteristics associated with receiving ANSs

\begin{tabular}{|c|c|c|c|c|c|c|c|c|c|}
\hline \multirow[b]{2}{*}{ Variable ${ }^{*}$} & \multirow[b]{2}{*}{ Total sample } & \multicolumn{4}{|c|}{$\geq 1500 \mathrm{~g}$} & \multicolumn{4}{|c|}{$<1500 \mathrm{~g}$} \\
\hline & & Total & ANSs & No ANSs & $p$-value & Total & ANSs & No ANSs & $p$-value \\
\hline Antenatal care, $n / N(\%)$ & $\begin{array}{l}1773 / 2085 \\
(85.0)\end{array}$ & $\begin{array}{l}587 / 676 \\
(86.8)\end{array}$ & $\begin{array}{l}178 / 193 \\
(92.2)\end{array}$ & $\begin{array}{l}409 / 483 \\
(84.7)\end{array}$ & 0.009 & $\begin{array}{l}1186 / 1409 \\
(84.2)\end{array}$ & $\begin{array}{l}668 / 729 \\
(91.6)\end{array}$ & $\begin{array}{l}518 / 680 \\
(76.2)\end{array}$ & $<0.001$ \\
\hline $\begin{array}{l}\text { Maternal hypertension, } \\
n / N(\%)\end{array}$ & $\begin{array}{l}552 / 2100 \\
(26.3)\end{array}$ & $\begin{array}{l}126 / 685 \\
(18.4)\end{array}$ & $\begin{array}{l}54 / 197 \\
(27.4)\end{array}$ & $\begin{array}{l}72 / 488 \\
(14.8)\end{array}$ & $<0.001$ & $\begin{array}{l}426 / 1415 \\
(30.1)\end{array}$ & $\begin{array}{l}260 / 732 \\
(35.5)\end{array}$ & $\begin{array}{l}166 / 683 \\
(24.3)\end{array}$ & $<0.001$ \\
\hline Maternal HIV, $n / N(\%)$ & $\begin{array}{l}648 / 2101 \\
(30.8)\end{array}$ & $\begin{array}{l}227 / 688 \\
(33.0)\end{array}$ & $\begin{array}{l}53 / 197 \\
(26.9)\end{array}$ & $\begin{array}{l}174 / 491 \\
(35.4)\end{array}$ & 0.031 & $\begin{array}{l}421 / 1413 \\
(29.8)\end{array}$ & $\begin{array}{l}197 / 731 \\
(26.9)\end{array}$ & $\begin{array}{l}224 / 682 \\
(32.8)\end{array}$ & 0.015 \\
\hline Vaginal delivery, $n / N(\%)$ & $\begin{array}{l}718 / 2101 \\
(34.2)\end{array}$ & $\begin{array}{l}237 / 688 \\
(34.4)\end{array}$ & $\begin{array}{l}52 / 196 \\
(26.5)\end{array}$ & $\begin{array}{l}185 / 492 \\
(37.6)\end{array}$ & 0.006 & $\begin{array}{l}481 / 1413 \\
(34.0)\end{array}$ & $\begin{array}{l}204 / 730 \\
(27.9)\end{array}$ & $\begin{array}{l}277 / 683 \\
(40.6)\end{array}$ & $<0.001$ \\
\hline Multiple gestation, $n / N(\%)$ & $\begin{array}{l}433 / 2100 \\
(20.6)\end{array}$ & $\begin{array}{l}172 / 689 \\
(25.0)\end{array}$ & $\begin{array}{l}51 / 197 \\
(25.9)\end{array}$ & $\begin{array}{l}121 / 492 \\
(24.6)\end{array}$ & 0.723 & $\begin{array}{l}261 / 1411 \\
(18.5)\end{array}$ & $\begin{array}{l}114 / 729 \\
(15.6)\end{array}$ & $\begin{array}{l}147 / 682 \\
(21.6)\end{array}$ & 0.004 \\
\hline
\end{tabular}

significantly associated with ANS exposure. However, when the total group was divided according to birth weight $<1500 \mathrm{~g}$ and $\geq 1500 \mathrm{~g}$, late-onset sepsis, RDS, CPAP and surfactant therapy were no longer significant. This indicates that birth weight was a major confounding factor.

\section{Neonates $\geq 1500 \mathrm{~g}$ (Tables 2 and 3 )}

In the subgroup weighing $\geq 1500 \mathrm{~g}$, there were $197 / 691$ neonates (28.5\%) exposed to ANSs and a survival rate of 674/690 (97.7\%). There was a significantly lower mean birth weight $(p=0.002)$ and mean gestational age $(p<0.001)$ in neonates exposed to ANSs compared with those who were not exposed. It was observed that neonates exposed to ANSs had a longer duration of stay than those who were not exposed $(p=0.002)$. There was no significant difference between exposure to ANSs and RDS, IVH, NEC, PDA, early- and late-onset sepsis, and other neonatal characteristics. The variables most significantly associated with ANSs using logistic regression (Table 4$)$ were antenatal care $(p=0.015)$, vaginal delivery $(p=0.043)$, maternal hypertension $(p=0.002)$, birth weight $(p=0.034)$ and gestational age $(p<0.001)$. Maternal HIV and duration of stay were not significant after logistic regression.

\section{Neonates $<1500 \mathrm{~g}$ (Tables 2 and 3 )}

In the subgroup weighing $<1500 \mathrm{~g}$, there were $733 / 1418$ neonates exposed to ANS (53.1\%) and a survival rate of $1075 / 1418$ (75.8\%). Neonates exposed to ANSs were significantly less likely to have a PDA $(p=0.015)$, to have an IVH $(p=0.049)$, and to require resuscitation at birth $(p<0.001)$. There was a significant association between exposure to ANSs and increased survival $(p<0.001)$. It was also observed that neonates exposed to ANSs had a longer duration of stay in hospital than those who were not exposed $(p=0.001)$. There was no significant association between ANS and RDS, NEC, early- and lateonset sepsis and other neonatal characteristics. The variables most significantly associated with ANSs using logistic regression (Table 5) were antenatal care $(p<0.001)$, vaginal delivery $(p=0.038)$, multiple gestation $(p<0.001)$, resuscitation at birth $(p=0.001)$, PDA $(p=0.001)$ and duration of stay $(p=0.012)$. Variables that were not significant after logistic regression were conventional ventilation, IVH, outcome, maternal hypertension and HIV.

\section{Discussion}

The current study showed ANS coverage of $44.1 \%$ at CMJAH. This figure has increased since 1993 , when coverage was only $20 \%,{ }^{[8]}$ but it is lower than in other LMICs, which according to the WHO Multicountry Survey on Maternal and Newborn Health had an average coverage of $52 \%{ }^{[15]}$ ANS coverage at CMJAH is substantially lower than the average of $80 \%$ in high-income countries (HICs). ${ }^{[1]}$ An increase in gestational age was associated with decreased exposure to ANS. This trend is also noted in HICs, where ANS coverage decreases by more than half at 34 weeks compared with coverage at 24 - 30 weeks. ${ }^{\left[{ }^{[6]}\right]}$ The reasons for this are not clear. It may represent inaccurate gestational age estimation or reduced attention to 'moderately preterm' babies. ${ }^{[16]}$

Our study showed surprising results when the total sample was analysed, as certain results were significant in the total sample and not significant in either group when stratified by birth weight. This is because more small babies (51.7\%), than larger babies (28.5\%), were exposed to ANSs, showing that birth weight is a confounding factor.

In our study, vaginal delivery was associated with decreased ANS use. This is true in other LMICs and HICs. ${ }^{[15,16]}$ The main reason for this decrease in ANS use is mothers presenting in advanced labour, giving no opportunity for ANSs to be provided..$^{[8]}$ We also found that unbooked mothers were significantly less likely to receive ANSs. Lack of antenatal care is consistently associated with reduced ANS coverage in LMICs and HICs. ${ }^{[15,16]}$ This is a difficult problem to address, as it is probably associated with low socioeconomic status and poor maternal education and is a point for improvement.

In our study, maternal hypertension had an association with increased ANS use. This is probably because mothers with hypertension are followed up regularly during the antenatal period and are usually admitted if preterm delivery is necessary. The decreased ANS exposure associated with maternal HIV infection was an interesting observation, but cannot be explained by our study. It was, however, not significant on logistic regression.

We found no association between ANS exposure and RDS, NEC, early-onset sepsis or need for respiratory support. This contradicts the Cochrane review ${ }^{[5]}$ on the effect of ANSs on preterm neonates, but Pattinson et al. ${ }^{[12]}$ also showed no statistical significance in RDS, NEC, early-onset sepsis and need for ventilation in their study in SA. It must be pointed out, however, that the neonates in Pattinson et al's study had a higher mean birth weight than those in our study.

We found that neonates exposed to ANSs were less likely to have a PDA than those who were not exposed. This supports Salhab et al.'s ${ }^{[6]}$ finding of a beneficial effect of ANSs on PDA. Possible mechanisms for this are a steroid effect on prostaglandin synthesis, reduced sensitivity of the ductal muscle to prostaglandin $\mathrm{E}_{2}$, and increased activity of the prostaglandin $E_{2}$ inactivation enzyme. ${ }^{[6]}$ 


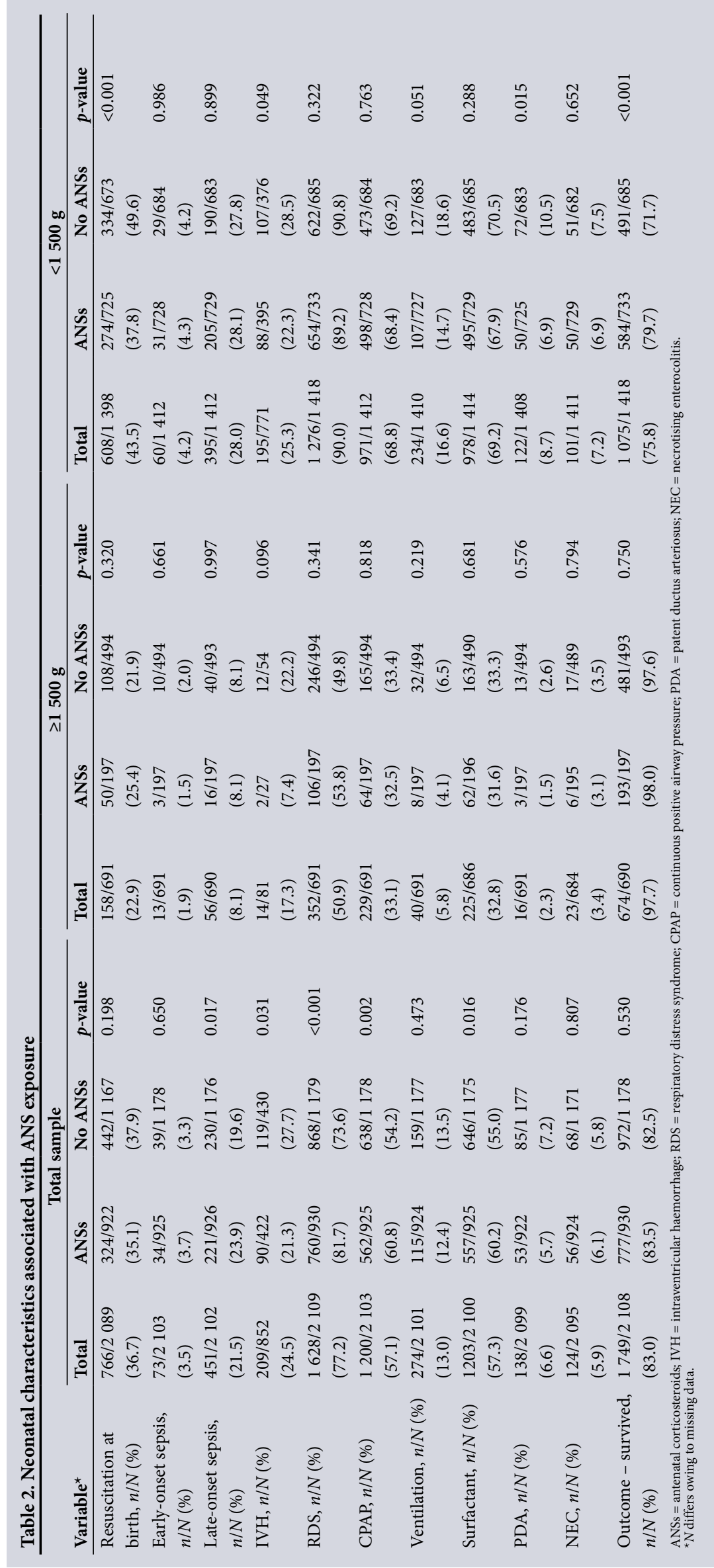

Our study demonstrated a significant association between ANS exposure and survival in neonates weighing $<1500 \mathrm{~g}$. This finding is in agreement with Pattinson et al. ${ }^{[12]}$ and the Cochrane review on the effect of ANSs on preterm neonates, ${ }^{[5]}$ but contrasts with Althabe et al'. ${ }^{[11]}$ observation of an increase in neonatal mortality in LMICs with the upscaling of ANSs and differs from the conclusions of Azad and Costello, ${ }^{[10]}$ who questioned whether ANSs improve neonatal mortality. Our study also showed an association between ANS exposure and reduced IVH. This association was only significant in neonates weighing $<1500 \mathrm{~g}$ and supports the findings of the Cochrane review $^{[5]}$ that ANS exposure reduces the risk of IVH.

Our study demonstrated a longer duration of stay in both weight groups, <1 500 and $\geq 1500 \mathrm{~g}$, for neonates exposed to ANSs compared with those who were not exposed. This interesting observation could be explained by a significantly higher survival rate in the ANS-exposed group, and the fact that the lower mean birth weight (1 $236 \mathrm{~g}$ ) in this group than in the unexposed group (1 $336 \mathrm{~g}$ ) meant that it took longer for the babies who had been exposed to reach $1600 \mathrm{~g}$, the discharge weight at CMJAH. Most deaths at CMJAH occur within the first 7 days of life.

There were no associations between ANS exposure and improved neonatal outcomes in neonates weighing $\geq 1500 \mathrm{~g}$ in our study. This supports Azad and Costello's ${ }^{[10]}$ observation that the use of ANSs is of no benefit after 33 weeks' gestation in LMICs.

\section{Study limitations}

This was a retrospective study, and there was low ANS coverage. The number and timing of ANS doses were not known. We can only comment on associations and cannot address effects of ANSs in LMICs. Since birth weight instead of gestational age was used, babies with intrauterine growth restriction may have been included in our sample.

\section{Conclusions}

Preterm birth is associated with multiple complications. There have recently been questions on the efficacy of ANSs in LMICs. The present study showed an association between ANSs and increased survival, decreased PDA and decreased IVH in neonates weighing $<1500$ g. However, there was no association between ANSs and RDS, NEC or sepsis, and there were no associations with improved neonatal outcome in neonates weighing $\geq 1500 \mathrm{~g}$. This suggests a review of the birth weight cut-off point for 
Table 3. Continuous variables associated with ANS exposure

\begin{tabular}{|c|c|c|c|c|c|c|}
\hline \multirow[b]{2}{*}{ Variable } & \multicolumn{3}{|c|}{$\geq 1500 \mathrm{~g}$} & \multicolumn{3}{|c|}{$<1500 \mathrm{~g}$} \\
\hline & ANSs & No ANSs & $p$-value & ANSs & No ANSs & $p$-value \\
\hline Birth weight (g), mean (SD) & $1633.8(84.7)$ & $1656.8(88.2)$ & 0.002 & $1129.7(234.2)$ & $1105.7(250.6)$ & 0.063 \\
\hline Gestational age (wk), mean (SD) & $32.0(1.6)$ & $32.7(2.3)$ & $<0.001$ & $29.1(2.3)$ & $29.0(2.8)$ & 0.531 \\
\hline Duration of CPAP (d), median (IQR) & $1(0)$ & $1(1)$ & 0.164 & $2(3)$ & $2(2)$ & 0.440 \\
\hline Duration of ventilation (d), median (IQR) & $8(13)$ & $4(3)$ & 0.069 & $5(9)$ & $5(6)$ & 0.608 \\
\hline Duration of stay (d), median (IQR) & $10(9)$ & $9(9)$ & 0.002 & $30(28)$ & $26(31)$ & 0.001 \\
\hline
\end{tabular}

Table 4. Logistic regression for significant variables in neonates weighing $\geq 1500$ g exposed to ANSs

\begin{tabular}{llll}
\hline Variable & OR & 95\% CI & $p$-value \\
\hline Antenatal care & 2.132 & $1.157-3.921$ & 0.015 \\
Vaginal delivery & 0.656 & $0.436-0.987$ & 0.043 \\
Maternal hypertension & 2.008 & $1.305-3.096$ & 0.002 \\
Birth weight & 0.998 & $0.996-1.000$ & 0.034 \\
Gestational age & 0.826 & $0.754-0.906$ & $<0.001$ \\
ANSs = antenatal corticosteroids; OR = odds ratio; CI = confidence interval.
\end{tabular}

Table 5. Logistic regression for significant variables in neonates weighing $<1500 \mathrm{~g}$ exposed to ANSs

\begin{tabular}{llll}
\hline Variable & OR & 95\% CI & p-value \\
\hline Antenatal care & 3.086 & $1.953-4.878$ & $<0.001$ \\
Vaginal delivery & 0.705 & $0.508-0.980$ & 0.038 \\
Multiple gestation & 0.493 & $0.334-0.728$ & $<0.001$ \\
Resuscitation at birth & 0.581 & $0.427-0.791$ & 0.001 \\
PDA & 0.462 & $0.290-0.736$ & 0.001 \\
Duration of stay & 1.009 & $1.002-1.016$ & 0.012 \\
ANSs = antenatal corticosteroids; PDA = patent ductus arteriosus; OR = odds ratio; \\
CI = confidence interval.
\end{tabular}

issuing ANSs in our setting, and perhaps indicates a need for a randomised controlled trial on the effects of ANSs in LMICs.

Acknowledgements. None.

Author contributions. AL conceptualised and designed the study, collected data, carried out data analysis, drafted the initial manuscript, revised the manuscript and approved the final manuscript; DEB conceptualised and designed the study, assisted with data analysis, reviewed and revised the manuscript, and approved the final manuscript; TR conceptualised and designed the study, reviewed and revised the manuscript, and approved the final manuscript; and TC reviewed and assisted with data analysis, reviewed and revised the manuscript, and approved the final manuscript. Funding. None.

Conflicts of interest. None.

1. Lawn J, Cousens S, Zupan J. 4 million neonatal deaths: When? Where? Why? Lancet 2005;365(9462):891900. https://doi.org/10.1016/s0140-6736(05)71048-5

2. Statistics South Africa. Millennium Development Goals 4: Reduce child mortality. Pretoria: SSA, 2015.

3. Velaphi S. Reducing neonatal deaths in South Africa - are we there yet, and what can be done? S Afr Child Health 2012;6(3):67-71. https://doi.org/10.7196/SAJCH.493

4. Liggins G, Howie R. A controlled trial of antepartum glucocorticoid treatment for prevention of the respiratory distress syndrome in premature infants. Pediatrics 1972;50(4):515-525.

5. Roberts D, Dalziel S. Antenatal corticosteroids for accelerating fetal lung maturation for women at risk of preterm birth. Cochrane Database Syst Rev 2006, Issue 3. Art. No.: CD004454. https://doi org/10.1002/14651858.cd004454.pub2

6. Salhab W, Hynan L, Perlman J. Partial or complete antenatal steroids treatment and neonatal outcome in extremely low birth weight infants < 1000g: Is there a dose-dependent effect? J Perinatol 2003;23(8):668672. https://doi.org/10.1038/s.jp. 7211007

7. Mwansa-Kambafwile J, Cousens S, Hansen T, Lawn J. Antenatal steroids in preterm labour for the Mwansa-Kambafwile J, Cousens S, Hansen T, Lawn J. Antenatal steroids in preterm labour for the
prevention of neonatal deaths due to complications of preterm birth. Int J Epidemiol 2010;39(Suppl prevention of neonatal deaths due to complica
1):i122-i133. https://doi.org/10.1093/ije/dyq029

8. Ballot D, Ballot N, Rothberg A. Reasons for failure to administer antenatal corticosteroids in preterm . Ballot D, Ballot N, Rothberg A. Reasons for
labour. S Afr Med J 1995;85(10):1005-1007.

labour. S Afr Med J 1995;85(10):1005-1007. National Institute for Health and Clinical Excellence. Preterm labour and
2015. https://www.nice.org.uk/guidance/ng25 (accessed 16 April 2017).

10. Azad K, Costello A. Extreme caution is needed before scale-up of antenatal corticosteroids to reduce preterm deaths in low-income settings. Lancet Global Health 2014;2(8):e191-e192. https://doi org/10.1016/s2214-109x(14)70020-8

11. Althabe F, Belizán L, McClure E, et al. A population-based, multifaceted strategy to implement antenatal corticosteroid treatment versus standard care for the reduction of neonatal mortality due to preterm birth in low-income and middle-income countries: The ACT cluster-randomised trial. Lancet 2015;385(9968):629-639. https://doi.org/10.1016/s0140-6736(14)61651-2

12. Pattinson R, Makin J, Funk M, et al. The use of dexamethasone in women with preterm premature rupture of membranes - a multicentre, double-blind, placebo-controlled, randomised trial. S Afr Med J 1999;89(8):865-870.

13. Jardine C, Ballot D. The use of nasal CPAP at the Charlotte Maxeke Johannesburg Academic Hospital S Afr J Child Health 2015;9(2):45-48. https://doi.org/10.7196/SAJCH.859

14. Harris P, Taylor R, Thielke R, Payne J, Gonzalez N, Conde J. Research Electronic Data Capture (REDCap) - a metadata-driven methodology and workflow process for providing translational research informatics support. J Biomed Inform 2009;42(2):377-381. https://doi.org/10.1016/j.jbi.2008.08.010

15. Vogel J, Souza J, Gülmezoglu A, et al. Use of antenatal corticosteroids and tocolytic drugs in preterm births in 29 countries: An analysis of the WHO Multicountry Survey on Maternal and Newborn Health Lancet 2014;384(9957):1869-1877. https://doi.org/10.1016/s0140-6736(14)60580-8

16. Lee H, Lyndon A, Blumenfeld Y, Dudley R, Gould J. Antenatal steroid administration for premature infant in California. Obstet Gynecol 2011;117(3):603-609. https://doi.org/10.1097/aog.0b013e31820c3c9b

Accepted 4 May 2017. 